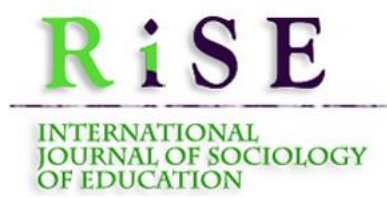

Hipatia Press

www.hipatiapress.com

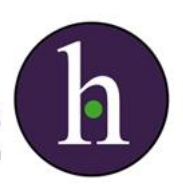

Instructions for authors, subscriptions and further details:

http://rise.hipatiapress.com

\title{
Programas de Orientación a Mujeres para la Inserción en el Mercado Laboral: De los Fundamentos Epistemológicos a la Transformación Social
}

Carmen de la Mata ${ }^{1}$

Bárbara Luque Salas ${ }^{1}$

1) Universidad de Córdoba, Spain

Date of publication: October $25^{\text {th }}, 2018$

Edition period: October 2018-February 2019

To cite this article: De la Mata, C., Luque Salas, B. (2018). Programas de Orientación a Mujeres para la Inserción en el Mercado Laboral: De los Fundamentos Epistemológicos a la Transformación Social, International Journal of Sociology of Education, 7(3), 212-236. doi:

10.17583/rise.2018.3442

To link this article: http://dx.doi.org/10.17583/rise.2018.3442

\section{PLEASE SCROLL DOWN FOR ARTICLE}

The terms and conditions of use are related to the Open Journal System and to Creative Commons Attribution License (CC-BY) 


\section{Women Orientation Program for the Insertion in the Labour Market: From Epistemological Basics to Social Transformation}

Carmen de la Mata

University of Córdoba
Bárbara Luque Salas

University of Córdoba

(Received: 12 April 2018; Accepted: 22 May 2018; Published: 25 October 2018)

\section{Abstract}

This manuscript shows the importance of the theoretical feminist corpus within employment orientation itineraries. Since the end of the 90's orientation programs are being implemented whose objective is to promote the participation in the labour market and to drive their professional careers. These performances are put in place as corrective measures at the obstacles women find at the beginning and during their careers paths. Those actions are called "With gender perspective" in order to meaning them and to be distinguished from those who don't have that compensatory character. What value does gender perspective add to these insertion itineraries? These processes training seek social transformation, reducing the labor market participation gender gap respect to employment, incorporating in an innovative way the epistemological foundations of feminist critical theory that will be presented throughout this article.

Keywords: educational counselling, career development, education, gender 


\section{Programas de Orientación a Mujeres para la Inserción en el Mercado Laboral: De los Fundamentos Epistemológicos a la Transformación Social}

Carmen de la Mata

University of Córdoba
Bárbara Luque Salas

University of Córdoba

(Recibido: 12 Abril 2018; Aceptado: 22 Mayo 2018; Publicado: 25 Octubre 2018)

\section{Resumen}

Este manuscrito presenta la importancia del corpus teórico feminista en la práctica de la orientación para la mejora de la empleabilidad de las mujeres. Desde finales de la década de los años 90 del siglo pasado se están implementando programas de orientación que tienen por objetivo fomentar la participación en el mercado laboral e impulsar sus carreras profesionales. Estas actuaciones se ponen en marcha como medidas correctoras ante los obstáculos que las mujeres encuentran en el inicio y el desarrollo de su trayectoria laboral, y se señalan con la denominación con perspectiva de género para significarlas y distinguirlas de aquellas que no tienen ese carácter compensatorio. ¿Qué valor añade la denominación con perspectiva de género a estos itinerarios de inserción? Estos procesos de formación tutorizada buscan la transformación social, reduciendo la brecha de participación en el mercado laboral entre mujeres y hombres con respecto al empleo, incorporando de manera innovadora los fundamentos epistemológicos de la teoría crítica feminista que se expondrán a lo largo de este artículo.

Palabras clave: orientación educativa, orientación profesional, género, educación 
finales del siglo XX, y como consecuencia del Libro Blanco de la Comisión Europea sobre crecimiento, competitividad y empleo (1993), comienzan a desarrollarse iniciativas que tienen como objetivo fomentar la creación de puestos de trabajo para las personas con mayores dificultades: mujeres, menores sin cualificación, colectivos desfavorecidos o personas en riesgo de exclusión social y personas con discapacidad. En este escenario muchas entidades públicas y privadas ponen en marcha programas para abordar las dificultades de las mujeres en su acceso al mercado laboral y, para ello, se emprenden actuaciones que se enfocan desde diversas líneas: incrementar el acceso al empleo a través de medidas de formación, fomentar el acceso a trabajos de mayor cualificación y a puestos de liderazgo e incentivar la creación de pequeñas empresas o del propio empleo, entre otras. En esta apuesta por fomentar el acceso al empleo de las mujeres se comienzan a diseñar programas específicos que incluyen tutoría individual y grupal, formación, sesiones de apoyo o congresos con diferentes contenidos (seminarios de empleo, ferias de emprendimiento o eventos para creación de redes profesionales, desayunos de trabajo, etc.). A estos programas que se diseñan con el fin de apoyar a las mujeres que tienen dificultades de empleabilidad relacionadas con desventajas sobrevenidas por consecuencia de su papel social se les identifica incluyendo en su denominación con perspectiva de género o con enfoque de género'. Esta manera de significarlos deriva de su característica esencial: incluir el género como instrumento de trabajo y análisis para emprender cualquier tipo de aproximación a la realidad.

Estas iniciativas se ponen en marcha amparadas por el desarrollo que la epistemología feminista aporta a las distintas disciplinas científicas y con ello a sus estudios científicos. Los estudios de las mujeres como se comenzaron denominando o estudios de género tuvieron una evolución muy significativa a partir de los años 70 , gracias a la incorporación de las mujeres a la educación y a la ciencia como estudiantes, profesoras o científicas (Flecha, 2005; Barberá y Cala, 2008a). Esta expansión redundó en la praxis social a través del desarrollo programático de actuaciones dirigidas a impulsar a las mujeres a posiciones más igualitarias con respecto a los hombres en la sociedad. 
RISE - International Journal of Sociology of Education, 7(3) 215

\section{Marco Teórico}

\section{La Categoría Género en Relación con el Empleo}

Introducir el género como herramienta de trabajo en los procesos de inserción laboral facilita un análisis más crítico y descriptivo de la realidad. La palabra sexo nos remite a lo biológico, y el género, como categoría, nos hace entender que hay características socialmente atribuidas a las personas en función de esa biología o genitalidad. El empleo de ambos conceptos permite una radiografía de la diferente posición que ocupan en la cultura laboral ambos sexos y mejoras en el ejercicio de la orientación sociocomunitaria. La falta de equidad entre mujeres y hombres no es natural, y no tiene relación con los componentes biológicos sino con aspectos culturales. Introducir esta categoría nos desvela la atribución de características diferentes y polarizadas con las que se identifica a las personas: a las mujeres se les han exigido aquellas relacionadas con la reproducción (el amor, la abnegación, la bondad) y a los hombres, las relacionadas con la producción (el dinero, la competencia, el interés económico).

Durante la infancia y la vida adulta se asimilan por socialización diferenciada: valores, símbolos, creencias y formas de comportamiento ligadas a estas características que se relacionan con la identidad sexual. Esto tiene una repercusión en todas las esferas de la vida: biológica, psicológica, social y laboral, que se relacionan indefectiblemente con el ejercicio de la carrera profesional. El papel social atribuido a las mujeres se vincula con funciones reproductivas y se conecta al ámbito privado y del hogar; en cambio, el rol social del hombre se relaciona con funciones de gestión pública y de obtención de bienes pecuniarios, teniendo la responsabilidad del sustento económico de la familia (Carrasco, 1998). Ambas funciones requieren una gestión de espacios y tiempos diferentes. El espacio de la familia requiere un uso del tiempo muy distinto del que se necesita para el desenvolvimiento en el ámbito público, en el que el desarrollo de las tareas permite una distribución del tiempo diacrónica y discontinua. Sin embargo, el 'cuidado' aboca a quien lo realiza a una utilización del tiempo mucho más circular y sincrónica, en la que las tareas se superponen. Estas circunstancias alejan a las mujeres de los requerimientos y de las dinámicas del mercado 


\section{De La Mata \& Luque-Inserción Laboral Género}

laboral y provoca mayores dificultades con respecto a los hombres para el mantenimiento del trabajo remunerado y/o la búsqueda del empleo.

Los estudios de género, utilizando estos elementos fundamentales en la teoría feminista, impulsaron un acercamiento a la realidad que maneja dos aspectos esenciales: la inclusión del género como categoría para el análisis y la observación del mundo, lo que ofrece una mirada de sospecha que se rebela contra el discurso androcéntrico. Cuando hablamos de incluir el género como categoría de análisis es inevitable remitirse a la teoría o sistema sexo-género y con ello, hacer referencia a cómo, en torno a esta, se desarrolla un cuerpo de conocimiento que explica una visión de la vida de las mujeres y de la existencia humana, en general, más acertada. Aunque en la actualidad se revisan algunos elementos para que no resulten opacos en su entendimiento de una realidad en la que la variable sexo-género es mucho más compleja que hace años. Ambos términos han permitido esclarecer y visibilizar la posición diferenciada que ocupan las mujeres y hombres en la estructura social. La conjunción de estos dos elementos conduce a una mirada que se ha denominado con enfoque/perspectiva de género y nos devuelve cómo ambos sexos se sitúan de forma desigual en la organización social, siendo los campos de actuación diferentes, con funciones y con expectativas sociales distintas. Esta perspectiva ha permitido entender el género como un principio que ordena a las personas según su sexo y a abordar, a través de él, un análisis de los acontecimientos más contextualizado. Y, además, explicar los marcos políticos que sustentan y mantienen la diferencia, mediante un análisis hacia arriba de las instituciones (Harding, 2012, p. 50) que justifican y perpetúan los desequilibrios en la carrera laboral de mujeres y hombres.

De este modo, el género desvela cómo la distribución de roles tradicionales se convierte en un principio que determina la posición que ocupan las mujeres ante los recursos y explica las consecuencias que esto tiene en su carrera profesional. La trayectoria laboral de las mujeres manifiesta dificultades relacionadas con aspectos que derivan de esta desigualdad. Estudios realizados desde diferentes disciplinas (Ramos, Barberá y Sarrió, 2003; Gálvez, 2004; Instituto de la Mujer, 2005) manifiestan la existencia de barreras externas que se asocian a:

- Una mayor resistencia de las empresas y agentes empleadores a la inclusión de las mujeres en sus plantillas de trabajo por el 
menosprecio a la experiencia femenina en el mercado laboral.

- Una menor experiencia de las mujeres en aquellas disciplinas que tienen que ver con áreas como la gestión, la economía y la tecnología.

- Dificultades para encontrar recursos y apoyos que permitan la compatibilización entre el trabajo doméstico y el remunerado.

- Una menor disponibilidad crediticia debido a que las entidades financiadoras imponen intereses más altos y mayor cantidad de avales.

Estas barreras se relacionan con una mayor precariedad salarial, participación discontinua en el mercado de trabajo y segregación laboral, y condicionan los beneficios sociales que permiten una vida óptima.

Existen barreras que afectan a otros aspectos relacionados con la parte intrínseca del individuo y con la interiorización del mandato de género, la educación recibida y la devaluación sistemática que la sociedad hace de lo femenino o de las tareas asignadas a las mujeres. Distintas autoras (Kantor, 2002; Amoroso y Bosch, 2003; William, 2004; Coria, 2012) destacan dificultades internas relacionadas con:

- La falta de creencia en sí mismas por la carencia de mujeres que resulten modelos y referentes en su entorno.

- Una menor disponibilidad para la utilización del dinero familiar y dificultades para la negociación monetaria.

- Menor calidad de vida por la asunción en solitario de las cargas domésticas.

- Sentimientos de culpabilidad por el cuestionamiento de los roles tradicionales ante el desarrollo de la carrera profesional.

Estos últimos obstáculos de carácter interno se relacionan con el poco valor que se les atribuye a las tareas de cuidado en el sistema económico donde quedan invisibilizadas $\mathrm{y}$, en consecuencia, tienen una transferencia nula en el desarrollo de la carrera profesional (Carrasco, 2006). Las mujeres se enfrentan a dificultades relacionadas con la falta de autoestima, autoconcepto y a bajas expectativas ante el éxito, por la depreciación de aquellas capacidades que se adquieren con el ejercicio de las tareas de sostenimiento de la vida humana.

Los itinerarios de orientación al empleo con perspectiva de género contemplan estos aspectos y se ocupan, a través de su metodología, de que la 
identidad individual y profesional de las mujeres que quieren insertarse en el mercado laboral no quede desgajada o inservible en uno u otro escenario, privado o público.

\section{El Enfoque Feminista: Visiones e Influencias en el Proceso de Orientación}

La teoría crítica feminista hace emerger la necesidad de incluir una mirada que introduzca en el quehacer científico una visión que permita una construcción menos androcéntrica del conocimiento y menos esencialista de la ciencia, en un momento en el que se da un debate abierto sobre la neutralidad, universalidad y objetividad del método científico. Siguiendo a Blázquez (2012) el paradigma feminista, explica dos temas que resultan nodales en su desarrollo y a partir de los que se pueden entender las estrategias que guían la intervención social con perspectiva de género:

- Explica el género como un elemento que interacciona con otras categorías siendo un organizador clave de la vida social.

- Manifiesta la necesidad de que la generación de este cuerpo de conocimiento esté comprometido con la mejora de la vida de las mujeres, es decir, con la acción para hacer más igualitaria la sociedad.

De esta manera, todas las corrientes feministas proponen en relación con la producción de conocimiento, introducir una nueva mirada que integre los elementos sexo y género para generar un conocimiento más acertado y neutral que produzca mejoras sociales (Bartra, 2012). El considerar el género como categoría transversal a otras, sitúa el debate en la necesidad de revisar los marcos de pensamiento para acercar la ciencia al interés de las mujeres y a los temas que son de su preocupación y, así, romper con esa mirada que se había mantenido y que explicaba los acontecimientos desde una ciencia producida por varones situados en las posiciones de poder de la jerarquía social. Las diferentes aproximaciones feministas proponen una revisión del objeto científico y de los elementos sobre los que se construye: objetividad, racionalidad, universalidad y neutralidad. Además, instan a poner en marcha procedimientos que ofrezcan una investigación comprometida con la mejora de los intereses de las mujeres y que no solo evite el androcentrismo y el sexismo, sino que fomente avances en la igualdad entre mujeres y hombres. 
Aunque las posturas feministas sean plurales, todas ellas confluyen en estos dos postulados que constituyen los ejes principales del enfoque. Sin embargo, a partir de aquí, se producen distintos acercamientos desde los que debatir sobre el conocimiento, la metodología y el método científico. Estos han fomentado una producción extensa de elementos sobre los que reflexionar y en los que se ha fundamentado la intervención con enfoque de género.

Las teorías del punto de vista. Estas formulaciones hacen una crítica al marco epistemológico tradicional, explicando que este había desarrollado su conocimiento ignorando la perspectiva de las mujeres y, por este motivo, la ciencia y el conocimiento generado por el método científico no ha sido neutral sino sesgado. Estas teorías desvelan cómo la ciencia se había construido desde la perspectiva de los hombres y abordan el 'ser mujer' como una perspectiva epistemológicamente privilegiada. Las autoras que defienden estos postulados, Nancy Hartsock (1990), Evelyn Fox Keller (1985) y Sandra Harding (1996) expresaron que las mujeres por su posición en la estructura social tienen una visión particular del mundo que no ha sido expuesta ni escuchada por la ciencia. Las teorías del punto de vista subrayan que el conocimiento es 'situado' en tanto que los sujetos que investigan o son investigados están ligados a una biografía y a unas coordenadas histórico-contextuales. Las mujeres, por estar ubicadas en las posiciones ligadas a las tareas de la reproducción, poseen una ventaja situacional, dada la experiencia que les brinda la práctica del cuidado y el sostenimiento de la familia. Blázquez (2012) explica que Nancy Hartsock insiste en que:

No importa la edad, la etnia, la raza sino que lo relevante es que todas las mujeres hacen trabajo de mujeres al ser las responsables institucionalmente de producir seres humanos, lo que constituye un punto de vista desde el que interpretar la realidad. (p.30)

Las representantes de estas teorías explican que el género, 'ser mujer', es un punto de vista más apto que otros. Y con esta idea lo proponen como coordenada para producir un conocimiento que permita visibilizar, modificar y mejorar la posición de las mujeres en la estructura social.

El posmodernismo feminista. Este modelo rechaza el anterior privilegio epistémico y explica que el conocimiento defendido por las teorías del punto de vista es otra manera de expresar un conocimiento 'situado' que no está 
exento de sesgo, y argumenta que esta perspectiva no es tal, en tanto que 'la mujer' no existe, pues no hay una mujer única que pueda representar la perspectiva, lo cual sería una concepción esencialista. El posmodernismo feminista expone la existencia de infinitas coordenadas (raza, clase social, cultura) y, en consecuencia, asegura la existencia de una 'inestabilidad de la identidad social' en la que los múltiples puntos de vista existentes y su variabilidad no permiten un conocimiento situado. Norma Blázquez (2012) propone una mirada:

Desde el aquí y ahora, porque solo esto conduce a una perspectiva en la que las personas no estén completamente atrapadas epistémicamente dentro de sus culturas, sino que en cambio pueden escoger pensar desde otras localizaciones; no hay una correspondencia estable entre individuo y género. (p.33)

El posmodernismo feminista representado por Haraway (1988) hace una crítica al concepto hegemónico 'mujer' porque entiende que no hay una sola manera de entenderse de tal modo, sin embargo, no niega el género como categoría, sino que lo fragmenta en infinitas perspectivas; el género es una categoría superior transversal a otras.

El empirismo feminista. Esta teoría defendida por Helen Longino (1990) y Lynn Nelson (1990) propone que la falta de objetividad y la presencia de sesgos ocurren por errores en el proceso científico y en la disciplina que requiere el método a seguir. El empirismo explica que la ciencia no es femenina ni masculina y que sus métodos o técnicas tampoco lo son. Así, sitúa todas las expectativas de liberar a la ciencia de la perspectiva situacional en el abordaje de un procedimiento científico riguroso; solo eso puede generar una ciencia libre del error producido por la organización sociocultural de género. Propone introducir en el método la socialización del conocimiento como alternativa para eliminar la desviación impuesta por la perspectiva o situación particular. La forma de lograr la objetividad consiste en asegurar la pluralidad de perspectivas, a través de maximizar la confrontación de subjetividades, la explicitación de los compromisos derivados de situaciones particulares (del interés político o personal) y la apertura al criticismo. Estos postulados exponen que es necesario para evitar los errores que producen las construcciones genéricas que el interés quede expreso y se acepte la crítica para evitar la mirada única. 
En nuestra opinión, las distintas aproximaciones epistemológicas proponen elementos de reflexión que alumbran la articulación de prácticas originales en otros campos de trabajo y, concretamente, en la mejora de la empleabilidad de las mujeres. Estos elementos son:

- La necesidad de que las prácticas que se implementen estén comprometidas con la transformación social.

- La necesidad de un replanteamiento de los postulados tradicionales para reequilibrar el valor de 'lo femenino'. Tal y como las teorías del punto de vista revelaron, el conocimiento no había sido neutro al mostrar la ocultación de la perspectiva de las mujeres.

Una invitación a democratizar el conocimiento para acceder a posturas que expliquen la realidad más acertadamente. Así, lo propone el empirismo, que explica que el acceso a conclusiones más idóneas se consigue teniendo en cuenta todas las subjetividades y opiniones como modo de reducir la perspectiva interesada. Asimismo, el posmodernismo se propone superar la categoría género para huir del binarismo mujer/hombre. Tras 'ser mujer' otras 'localizaciones' son posibles, y establece un acercamiento a la usuaria que profundice más allá de la posición genérica que ocupa.

\section{Metodología}

Los razonamientos científicos en torno al género han permitido un análisis que invita a utilizar planteamientos metodológicos que fomenten el empoderamiento de las mujeres y la construcción de unas relaciones más equilibradas entre mujeres y hombres y en la que lo femenino no esté devaluado. Estas reflexiones alumbran aspectos de carácter epistemológico, metodológico e instrumental que permiten esgrimir estrategias en el desarrollo de programas de orientación significándolos como diferentes a los ordinarios. Resumiremos a tres las claves utilizadas que consideramos necesarias para que un itinerario de inserción al empleo tenga perspectiva de género.

1.- Agentes implicados con los valores feministas que busquen la transformación social

Con independencia de los objetivos que marcan todos los procesos de orientación, quienes trabajan desde el enfoque de género se plantean 
eliminar desigualdades y fomentar procesos de cambio social entre los sexos. El valor que incorporan estos itinerarios tiene que ir más allá de trabajar de manera no sexista o androcéntrica, para introducir mejoras sociales. La transformación social implica una selección cuidadosa de los equipos de trabajo o agentes que desarrollan estos programas que tienen que tener experiencia en la detección del sexismo, pero también ser profesionales comprometidos con los valores feministas porque se convierten en figurasclaves en el proceso, debido a la responsabilidad que contraen en la mejora de la situación de la usuaria.

El trabajo técnico está comprometido por el interés de que la beneficiaria tome poder en lo personal y en lo social para hacer frente a su proyecto de inserción laboral. Por ello, las personas expertas en esta perspectiva se entrenan para detectar el sexismo y acometen un ejercicio de reflexión sobre sus propias creencias para tomar consciencia de los estereotipos de género que mantienen que pudieran sesgar su práctica.

Como se ha explicado, muchas mujeres tienen dificultades relacionadas con barreras internas y externas que se derivan de los mandatos de género en los que han sido educadas, por ello, la persona que guía la orientación establece sinergias que sirvan de estímulo al proyecto laboral, y, al tiempo, generen vínculos para explorar en lo personal y para poder imbricarlo con lo profesional. Por tanto, es una condición indispensable por parte de la persona que orienta saber crear un clima de confianza con la usuaria para hablar abiertamente sobre su proyecto y también de aquellos aspectos personales y familiares que pueden incidir en la constitución de su empresa o en el proceso de búsqueda de empleo.

Se necesita un perfil profesional comprometido con el interés feminista para poder desentrañar, junto a la usuaria, aquellos obstáculos que tengan origen en su posicionamiento como 'ser mujer' y saber darles cauce. Es ineludible para la realización de la tarea, valorar que la vida de las mujeres y sus carreras profesionales no transcurren de manera lineal, sino que están anudadas a otros escenarios y a otros vínculos, en los que son las responsables de los cuidados de otras personas. Y estas tareas las alejan o dificultan su acceso y permanencia en el mercado laboral.

En definitiva, los procesos de orientación con perspectiva de género detectan las dificultades a las que la usuaria pudiera tener que enfrentarse como consecuencia de su rol social y pone de manifiesto cómo estas pueden 
ser un obstáculo en su carrera laboral si no se corrigen. Hay que tener en cuenta que muchas de las tareas que se realicen en estos itinerarios no repercutirán en eliminar las dificultades con las que las usuarias se encuentran. Sin embargo, el compromiso técnico con los postulados de la epistemología feminista contribuye al empoderamiento de las usuarias (Ander-Egg, 1990) y a generar condiciones óptimas para su acceso al mundo laboral.

2.- Diseños que se replantean los postulados tradicionales como consecuencia de considerar el diferente acceso a los recursos de las mujeres con respecto a los hombres

La epistemología feminista explica la necesidad de poner en cuestión la neutralidad de la ciencia y la necesidad de revisar los postulados que esta ha propuesto, basándose en la idea de que el individuo no es un ser neutro y deslocalizado y, en consecuencia, ni la ciencia ni las acciones que este diseña lo son (Habermas, 1986; Steinmetz, 2005). Esto ha marcado los itinerarios de orientación con perspectiva de género, introduciendo algunas consideraciones en sus fases. Estos dispositivos no cuestionan los métodos de orientación tradicionales sino la posibilidad de que la concepción que subyace a su diseño no se ajuste a la realidad de toda la población, porque entienden que los agentes sociales y las personas usuarias son individuos con biografía y sus actuaciones se guían por un interés ligado a la posición genérica que ocupan en la estructura social. Al igual que se cuestionó la falta de representación de los intereses de las mujeres en la ciencia, aquí se hace un replanteamiento metodológico e instrumental que tiene en cuenta que el sujeto que emprende el proyecto está ubicado en la estructura social y familiar de una determinada manera que se relaciona con el hecho de ser mujer.

En la concepción del modelo desde el que orientar se considera de manera especial acometer el proceso de orientación teniendo en cuenta 'el género' como categoría de análisis, utilizando esta como herramienta de planificación de los servicios y elaboración de los instrumentos. El momento en el que se idean y organizan los servicios aborda todo lo que comporta el planeamiento de las líneas de acción, elaboración de los materiales de trabajo, la selección de personal que lo ejecutará, la difusión del servicio y la evaluación del programa llevado a cabo. Y debe acometerse desde un análisis que permita una respuesta real al diferente acceso a los recursos de 
las mujeres con respecto a los hombres y la incidencia que esto tiene en cómo plantean sus proyectos laborales y las ideas que los sustentan. En el diseño de estos itinerarios toma un papel primordial la asignación de las tareas vitales que la sociedad vuelca en las mujeres y que la ubican en escenarios de responsabilidad que redundan en cómo se utilizan los espacios y los tiempos. Fundamentado en esta argumentación, estos itinerarios se diseñan teniendo en cuenta las siguientes consideraciones de género: 
Tabla 1

Consideraciones que orientan el diseño de actuaciones para la inserción laboral con perspectiva de género

\begin{tabular}{|c|c|}
\hline $\begin{array}{c}\text { FASES DE UN PROCESO DE } \\
\text { ORIENTACIÓN PARA LA INSERCIÓN }\end{array}$ & $\begin{array}{c}\text { CONSIDERACIONES } \\
\text { METODOLÓGICAS QUE APORTAN LOS } \\
\text { ITINERARIOS DE ORIENTACIÓN A LA } \\
\text { INSERCIÓN }\end{array}$ \\
\hline \multicolumn{2}{|c|}{ 1.- ACOGIDA } \\
\hline $\begin{array}{l}\text { Fase de inicio que explica el proceso y en la que } \\
\text { se define el itinerario. }\end{array}$ & $\begin{array}{l}\text { Dar especial importancia a una ejecución que } \\
\text { imprima confianza a la usuaria y que facilite la } \\
\text { implicación y la motivación que exige el } \\
\text { proceso combinando el asesoramiento técnico y } \\
\text { personal. }\end{array}$ \\
\hline
\end{tabular}

\section{2.- RECOGIDA DE INFORMACIÓN Y ANÁLISIS}

Fase para analizar y valorar conjuntamente la trayectoria profesional y personal y el grado de empleabilidad o maduración de la idea empresarial.
Subrayar la importancia de las actuaciones dirigidas a mejorar la autoestima, la autoeficacia, la negociación y gestión del uso del tiempo familiar y laboral.

\section{3.- DISEÑO DEL ITINERARIO PERSONALIZADO DE INSERCIÓN}

Dibujo del proceso de orientación teniendo en cuenta los aspectos detectados en la fase anterior.
Reforzar las acciones colaterales que faciliten las redes de contactos, la visibilización y la identificación de saberes aprendidos para ajustar la idoneidad del proyecto de trabajo a los recursos con los que cuenta la usuaria.

\section{4.- DESARROLLO DEL ITINERARIO PERSONALIZADO DE INSERCIÓN}

Fase de seguimiento para resolver trámites puntuales o reorientar cuestiones nuevas que puedan surgir.
Mediar en la búsqueda de otros profesionales o dispositivos de apoyo que puedan promocionar o invertir en el proceso.

\footnotetext{
5.- FINALIZACIÓN Y EVALUACIÓN DEL ITINERARIO PERSONALIZADO DE INSERCIÓN

Valoración de la mejora de la empleabilidad y definición de nuevos objetivos si fuese necesario.
} 
3.- Utilización de técnicas que contemplan el acercamiento a las usuarias y la escucha profunda

El empirismo invita a compartir las perspectivas científicas como modo de evitar enfoques reduccionistas que desvirtúen un análisis congruente de los acontecimientos. Igualmente, el posmodernismo aboga por ir más allá del género para que este no se convierta en un cristal opaco que invisibilice aquellas posturas o posiciones menos reconocidas del 'ser mujer'. Estos distintos enfoques ponen de manifiesto la necesidad de huir de la unicidad y del binarismo en pro de aceptar la pluralidad. En coherencia con estas ideas, la implementación del proceso de orientación con perspectiva de género se realiza como un proceso guiado, cuyo desarrollo tiene en cuenta y rescata la experiencia del sujeto (femenino), y lo hace situando al profesional y a la usuaria en un plano de igualdad, donde ambos aportan sus perspectivas y saberes al proyecto. Este modo de compartir el conocimiento y de explicitar intereses en la construcción del proyecto laboral pone en práctica esta estrategia porque democratiza el conocimiento y reduce la perspectiva única.

Los itinerarios de orientación utilizan métodos de trabajo que tienen como objetivo último acceder a las creencias y formas de entender el mundo que tienen las usuarias. Para ello, imprime a los procedimientos una clave de acercamiento a la perspectiva del sujeto que se relaciona con el análisis cualitativo y con técnicas propias de este paradigma. La epistemología feminista y la mayoría de sus estudios científicos se apropiaron de esta metodología por priorizar los aspectos subjetivos de la conducta humana, permitiendo la proximidad a las usuarias (Taylor y Bogdan, 2002).

En estos itinerarios se utiliza como técnica de trabajo la denominada 'entrevista en profundidad' definida por uno de sus máximos defensores, Johnson (2002) como aquella que "persigue un conocimiento a fondo de los valores personales y experiencia vivida". En la orientación con perspectiva de género los primeros contactos entre la persona que orienta y la usuaria se aborda a través de una entrevista de este tipo. Una vez que estas primeras citas se resuelven, el resto de las fases se pueden desarrollar combinando las asesorías para la consulta de temas puntuales o para trabajar contenidos estructurales a través de la entrevista (autoestima, autoeficacia) que son necesarios trabajar durante todo el proceso. 


\section{Tabla 2}

Aspectos que orientan la entrevista en profundidad

\section{ASPECTOS QUE ORIENTAN LA ENTREVISTA EN PROFUNDIDAD}

Analizar las razones que mueven a buscar un empleo.

Profundizar en los aspectos condicionantes.

Valorar los recursos propios que tiene la usuaria.

Detectar factores de desigualdad que pueden ser un condicionante para la inserción.

Profundizar en las expectativas y necesidades de la usuaria.

Motivar y reforzar el proceso y estrategias instrumentales con trabajo individual y trabajos de grupo una acción tutorial constante.
¿Qué importancia tiene el empleo para ti? ¿Cómo crees que se siente la gente que tiene un empleo? Si tener un empleo significase pasar menos tiempo con tu familia ¿Cómo te sentirías? ¿Cómo afrontarías esto? ¿Has pensado en un empleo por cuenta propia o por cuenta ajena?

Para comenzar el proceso de búsqueda de empleo hay que saber de cuánto tiempo disponemos. ¿Qué haces un día normal de tu vida? ¿Tienes personas a tu cargo?

¿Qué días podríamos trabajar juntas? ¿Tienes algún apoyo que te ayude con esta tarea? ¿Cuánto tiempo tienes para dedicarle a este proyecto? ¿Es real ese tiempo con el que cuentas?

¿Cuál fue tu último empleo? ¿Te gustaba? ¿Qué ocurrió? ¿Estarías dispuesta a estudiar de nuevo? ¿Utilizas las redes sociales?

Actitudes y tiempos de las mujeres para emplearse: datos de convivencia, personas dependientes, recursos de apoyo, tiempos disponibles, movilidad, disponibilidad de vehículo, intereses, necesidades y expectativas.

Cuéntame que buscas en un empleo: tener éxito, ayudar a la familia, ganar un salario, mantener a la familia, realizarte profesionalmente, aprender, sentirte útil, tener más dinero, tomar decisiones propias, dirigir a otras personas.

A través de la entrevista en profundidad se buscan asimilaciones que refuercen a la usuaria en su proceso, transferencias entre las tareas realizadas en el ámbito privado para lograr que los saberes acumulados sean un punto de partida en la adquisición de competencias que el mercado de trabajo les va a requerir.

Elaboración propia a partir de los materiales UNEM (ERGA, 2008)

En las primeras citas es muy importante identificar las relaciones existentes entre la trayectoria personal de la usuaria y la idea de inserción profesional que posee, con objeto de identificar las competencias y apoyos que pueden favorecer el desarrollo del proyecto. Por ello, esta técnica se considera la más adecuada para esta finalidad, dado que tiene en cuenta al sujeto de 
manera integral tratando de comprender a las personas en su marco de referencia. Se busca un instrumento que en su aplicación tenga en cuenta la biografía de la persona, su pasado y su presente. A través de estas entrevistas, estos aspectos que se mencionan sirven de guía a la persona que orienta sin condicionar, son las beneficiarias las que construyen el proceso como sujetos de su propia experiencia (Ruiz-Olabuénaga, 2012). La figura que guía el proceso se sitúa en el mismo plano que la participante entendiendo sus dificultades, se busca mirar más allá de las creencias propias para interpretar el punto de vista desde el que las usuarias se encuentran al iniciar el proyecto.

Esta técnica pone de manifiesto que todas las perspectivas son valiosas. En este sentido, pluraliza el conocimiento porque es un instrumento que encuentra su validez en la proximidad que procura al mundo de las personas participantes (Flick, 2004; Ruiz-Olabuénaga, 2013).

\section{Resultados}

Los argumentos teóricos que propone la teoría crítica feminista sugieren la necesidad de repensar la perspectiva desde la que se ha acometido la investigación y, por tanto, a revisar el valor de sus productos. Estas tesis explican cómo el conocimiento se ha construido desde una visión masculina, en tanto que ha sido la mirada de los hombres la que ha dado contenido a qué se considera conocimiento científico sin contemplar a las mujeres, ni como sujeto ni como objeto de ciencia. Las claves metodológicas que se presentan muestran cómo estos fundamentos epistémicos subyacen en los itinerarios con perspectiva de género situando a la discente como sujeto de su propio aprendizaje y elaboran procesos que respetan qué tiene que decir la actora sobre el proyecto que va acometer. En estos itinerarios el currículum oculto, el adquirido por la asunción de las responsabilidades domésticas, es un elemento esencial. La experiencia en el desarrollo de las tareas de cuidado y el trabajo de las barreras internas o personales son relevantes para trabajar competencias que se puedan trasladar al mercado laboral y adquirir confianza en sí mismas y sus saberes. La perspectiva de la usuaria y el análisis feminista es lo que fundamenta su diseño. 


\section{RISE - International Journal of Sociology of Education, 7(3)}

\section{Tabla 3}

\section{Claves de trabajo en itinerarios de inserción laboral con enfoque de género.}

Elaboración propia a partir del manual Dispositivos de creación de Empresas: Protocolo de actuación desde un enfoque de género

CLAVES DE TRABAJO EN ITINERARIOS DE INSERCIÓN LABORAL CON ENFOQUE DE GÉNERO

FASES EN EL PROCESO DE ORIENTACIÓN PARA LA INSERCIÓN AL EMPLEO 1.- ACOGIDA

\begin{tabular}{ll} 
1.- ACOGIDA & \multicolumn{1}{c}{ Objetivos } \\
Informar a la usuaria del proceso y & $\begin{array}{c}\text { Claves metodológicas con perspectiva de género } \\
\text { Profundización en los aspectos que condicionan la actitud }\end{array}$ \\
$\begin{array}{l}\text { actuaciones que se ofrecerán: servicios } \\
\text { propios y de otros dispositivos de apoyo. }\end{array}$ & y los tiempos de las mujeres para emplearse relacionados \\
Conocer las necesidades, motivos y & Análisis de las razones que mueven a buscar un empleo: \\
expectativas ante el proceso y su proyecto. & deseo propio o necesidades del entorno. \\
$\begin{array}{l}\text { Acordar con la usuaria el proceso y establecer } \\
\text { el cronograma. }\end{array}$ & Búsqueda del compromiso de la usuaria con el proceso. \\
\hline
\end{tabular}

\section{2.- RECOGIDA DE INFORMACIÓN Y ANÁLISIS}

\section{Objetivos}

Valorar las variables de diagnóstico profesional que van a incidir en la planificación del proceso de orientación.

Analizar el perfil para establecer el itinerario.
Claves metodológicas con perspectiva de género Valoración de la diferente posición que ocupan las mujeres en el acceso a los recursos.

Detección de qué factores de desigualdad pueden ser un condicionante para la inserción.

Análisis de posibilidades y condicionamientos para adaptar el itinerario.

\section{3.- DISEÑO DEL ITINERARIO PERSONALIZADO DE INSERCIÓN}

\section{Objetivos}

Definir el objetivo a alcanzar y secuenciar las acciones que permitirán su alcance.

Mediar con otros agentes para facilitar el acceso a otros contactos.

Tomar decisiones y renovar los compromisos.

\section{Claves metodológicas con perspectiva de género}

Análisis de la coherencia de las acciones del proceso en relación con resultado esperado: prioridades, uso y disponibilidad de los recursos.

Análisis de las condiciones de movilidad, red de contactos, conocimiento del entorno laboral.

Capacitación para la comunicación, asertividad, iniciativa, autoestima, autoeficacia, búsqueda de recursos, estrategias de negoción y manejo de soluciones.

\section{4.- DESARROLLO DEL ITINERARIO PERSONALIZADO DE INSERCIÓN}

\section{Objetivos}

Proporcionar apoyo técnico y personal en el reajuste de las acciones y tiempos.
Claves metodológicas con perspectiva de género

Refuerzo de elementos como: autoconcepto, autoeficacia o corresponsabilidad.

Fortalecimiento de las estrategias instrumentales.

\section{FINALIZACIÓN Y EVALUACIÓN DEL ITINERARIO PERSONALIZADO DE INSERCIÓN}

\section{Objetivos}

Evaluar los resultados y el grado de consecución de los objetivos previstos.

Valorar las posibilidades de finalización o de redefinición del proceso.

\section{Claves metodológicas con perspectiva de género}

Evaluación del incremento de estrategias logradas a nivel personal y sociolaboral.

Analizar la mejora del posicionamiento en la estructura social de la usuaria.

Fundación Mujeres (2004), Materiales Unidades de Empleo de Mujeres (Erga, 
Del mismo modo que las teorías del punto de vista Harding (1996) invitan a hacer una ciencia que se acerque al objeto de estudio, este modelo de orientación utiliza técnicas y claves que se acercan a la usuaria, y entiende que una praxis social, que tenga la capacidad de situarse en el interés de las mujeres y acceder a sus interpretaciones, será más eficaz para producir cambios sociales. En consonancia con estas reflexiones, los itinerarios con enfoque de género invitan a cuestionar conceptos como el trabajo, la cualificación, el riesgo o la innovación empresarial, y métodos de trabajo, introduciendo en cada fase del proceso claves que mejoran la actividad realizada con las mujeres y fomentan el cambio social.

\section{Discusión}

Los itinerarios de inserción laboral proyectan en su implementación los elementos teóricos del feminismo, subrayando el valor que han tenido estos principios en los procedimientos de intervención práctica, esta argumentación está en consonancia con los trabajos de diversas autorías (García-Calvente, 2011; López et al. 2011; Denzin y Lincoln, 2013). Estos principios teóricos se explican en primer lugar, por la utilización como ventaja de la ubicación de las mujeres en la estructura social para buscar patrones que no habían sido reconocidos con anterioridad como importantes, y que, revisados desde una óptica no androcéntrica, aporten otro valor. En la misma línea, las teorías del punto de vista consideran que la localización de las mujeres en la estructura social es un punto privilegiado desde el que acercarse al análisis de la realidad. Autoras como Villariño y Cánnoves, (2000) y Nieto (2003) explican la importancia del papel social de las mujeres en el reconocimiento de necesidades y demandas sociales, que no se descubrirían desde otras perspectivas.

En segundo lugar, por la resignificación que promueven de los conocimientos aprendidos a través de la asunción de las responsabilidades familiares y domésticas, tal y como explican distintas autorías (Anker, 1997; Fundación Mujeres, 2004; Calvo y Álvarez, 2016) y los manuales de uso elaborados por ellas. En estos itinerarios se trabaja el valor que esta experiencia tiene de transferible en el proyecto profesional visibilizando la importancia de las tareas tradicionalmente desarrolladas por las mujeres. Y, por último, porque requiere de una asesoría comprometida con los valores 
que guían la intervención que sea capaz de dar valor a esta ubicación y fomentar la capacidad de empoderamiento de las usuarias. IronstoneCatterall (1998) explica cómo ser expertas de sus propias vidas les da voz. La transformación de lo aprendido y la instrumentalización de esos saberes aumenta la capacidad de las mujeres para tomar decisiones sobre su propia vida, tal y como la literatura científica ha demostrado (Rappaport, 1987; Romero, 1996; Velaz de Medrano, 2003, 2005).

\section{Conclusiones}

Los programas para mejorar la empleabilidad de las mujeres han utilizado la experiencia teórica acumulada por la comunidad feminista en la implementación de sus acciones. Fruto del debate, científicas y actoras sociales, han aplicado los postulados feministas y los métodos de trabajo que estos emplean, para implementar acciones de intervención social desde las que hacer una sociedad más igualitaria e impulsar a las mujeres en la estructura social a posiciones más equilibradas. Estas actuaciones con enfoque de género han utilizado los postulados de la teoría crítica feminista de manera original frente a las prácticas tradicionales de orientación para la inserción al mercado laboral. Sin embargo, en ocasiones, en la inercia de su desarrollo queda oculto el origen y la fundamentación de estas prácticas. Es necesario recordar los fundamentos que motivan estas prácticas para que cumplan los objetivos que las sustentan, de otro modo se corre el riesgo de que pierdan la fuerza que les imprime su génesis: el compromiso por el cambio social y por la mejora de un acceso equitativo de las mujeres a los recursos. Este artículo presenta los elementos del enfoque de la teoría crítica feminista como constitutivos de los itinerarios de inserción al empleo con enfoque de género. La finalidad es subrayar el valor de estas acciones y reconstruir los lazos existentes con los elementos teóricos del feminismo.

En esta tarea, se observa que las actuaciones para la mejora de la inserción laboral con enfoque de género:

- Rescatan el valor del conocimiento de las usuarias al utilizar sus experiencias para construir lo profesional mediante la traslación de las capacidades aprendidas en ámbito personal. Esta clave metodológica supone una restitución de los saberes de las mujeres que durante tanto tiempo han sido devaluados. 
- Impulsan cambios a nivel personal en las mujeres, que encuentran en ellas mismas saberes, que tienen un valor como herramienta en el mercado laboral del que ellas son poseedoras. La práctica social se sirve de los postulados que aportan las teorías del punto de vista para que los aspectos que conforman lo femenino tengan un valor en el mercado económico.

- Consiguen que las usuarias tomen conciencia a lo largo del proceso de orientación de las dificultades que afrontan por la posición desigual que mantienen con respecto a los hombres, teniendo esto un efecto multiplicador en su entorno.

- Promueven un replanteamiento de la actividad orientadora y del entendimiento del individuo y el entorno al proponer huir de posiciones binarias y "genéricas". Y lo hace sin dañar el valor que tienen las tareas reproductivas en el desarrollo social y en el mercado económico, invitando a su realización y revalorización.

Es necesario continuar con estas acciones específicas subrayando el carácter transformador de su práctica y entender su diseño desde la epistemología feminista, como elemento que asegura un marco mínimo de estrategias y que facilita un ejercicio coherente de la aplicación de la perspectiva de género.

\section{Referencias}

Adán, C. (2006). Feminismo y conocimiento: de la experiencia de las mujeres al ciborg. A Coruña: Espiral Maior.

Amoroso, M. I.; Bosch, A. (2003). Malabaristas de la vida: mujeres, tiempos y trabajos. Barcelona: Icaria.

Ander-Egg, E. (1990). Repasando la investigación-acción-participativa. México. DF: Editorial El ateneo.

Anker, R. (1997). La segregación profesional entre hombres y mujeres: repaso de las teorías. Revista internacional del trabajo, 116(3), 333370

Barberá, E. y Cala, M. J. (2008). Perspectiva de género en la psicología académica española. Psicothema, 20(2), 236-242.

Barberá, E. y Cala, M. J. (2008a). Desarrollo histórico en la investigación psicológica del enfoque de género. Revista de Historia de la Psicología, 29(3), 25-33. 
Batra, E. (2012). Acerca de la investigación y la metodología feminista. En N. Blázquez, F. Flores y M. Ríos (Coords.), Investigación feminista: epistemología, metodología y representaciones sociales (pp. 67-78). México DF: UNAM.

Blázquez, N. (2012). Epistemología feminista: temas centrales. En N. Blázquez, F. Flores y M. Ríos (Coords.), Investigación feminista: epistemología, metodología y representaciones sociales (pp. 21-38). México DF: UNAM.

Brush, C. (1997). Women-owned businesses: obstacles and opportunities. Journal of Development Entrepreneurship, 2(1), 1-24.

Cala, M. J. (2004). Metodología y procedimientos de análisis. En E. Barberá, Psicología y género (pp. 81-106). Madrid: Pearson Educación.

Calvo, C. y Álvarez, P. (2016). Guía para la creación de negocios rurales. Empoderamiento económico de las mujeres. Fundación Mujeres: Cáceres

Carrasco, C. (1998). Trabajo y empleo: apuntes para una discusión no androcéntrica. En A. Rodríguez, Reorganización del trabajo y el empleo de las mujeres (pp. 51-78). Alzira: Germania.

Carrasco, C. (2006). La paradoja del cuidado: necesario pero invisible. Revista de Economía Crítica, 5, 39-64.

Comisión de las Comunidades Europeas (1993). Libro Blanco de la Comisión Europea sobre crecimiento, competitividad y empleo: retos y pistas para entrar en el siglo XXI. Bruselas: Oficina de Publicaciones Oficiales de las Comunidades Europeas.

Coria, C. (2012). El sexo oculto del dinero. Barcelona: Red Ediciones. Denzin, N. y Lincoln, Y. (coords.) (2013). Las estrategias de investigación cualitativa. Barcelona: Gedisa, 2013.

Erga (2008). Unidades de Empleo para Mujeres: guía metodológica y de trabajo. Sevilla: Instituto Andaluz de la Mujer.

Flecha, C. (2005). La categoría género en los estudios feministas. En I. de Torres (coord.), Miradas desde la perspectiva de género: estudios de las mujeres (pp. 33-45). Madrid: Narcea.

Flick, U. (2004). Introducción a la investigación cualitativa. Madrid:

Morata. 
Fox-Keller, E. (1985). Reflection in gender and science. New Haven: Yale University Press.

Fundación Mujeres (2004). Dispositivos de creación de empresas: protocolo de actuación desde un enfoque de género. Asturias: Fundación del Metal.

Fundación Mujeres (2010). Igualdad de oportunidades entre mujeres y hombres en la actividad empresarial. Madrid: Instituto de la Mujer.

Gálvez, L. (2004). Logros y retos del análisis de género en la historia económica de la empresa. ICE Historia Empresarial, 812, 77-88.

García-Calvente, M. M. y Marcos, J. (2011). Construir salud, construir género: ¿por qué la investigación cualitativa es una metodología sensible al género? Feminismo/s, 18, 271-280.

Habermas, J. (1986). Conocimiento e interés. Madrid: Taurus.

Haraway, D. (1988). Situated knowledges: The science question in feminism and the privilege of partial perspective. Feminist studies, 14(3), 575599. doi: $10.2307 / 3178066$

Harding, S. (1986). The science question in feminism. New York: Ithaca. Harding, S. (1987). Feminism and methodology. Indiana: Indiana University Press.

Harding, S. (1996). Ciencia y feminismo. Barcelona: Morata.

Harding, S. (2012). ¿Una filosofía de la ciencia socialmente relevante?: argumentos en torno a la controversia sobre el punto de vista feminista. En N. Blázquez, F. Flores y M. Ríos (Coords.), Investigación feminista: epistemología, metodología y representaciones sociales (pp. 39-66). México DF: UNAM.

Hartsock, N. (1990). Foucault on power: a theory for women? Feminism/postmodernism, 162.

Instituto de la Mujer (2005). Autoempleo y actividades empresariales de las mujeres en España. Madrid: Ministerio de Trabajo y Asuntos Sociales.

Ironstone-Catterall, P. (1998). Feminist research methodology and women's health: a review of the literature. Toronto: National Network on Environments and Womens Health.

Johnson, J. M. (2002). In-depth interviewing. En J. Gubrium y J. Holstein (eds.), Handbook of qualitative research (pp. 103-119). Thousand Oaks, California: Sage. 
Kantor, P. (2002). Gender, microenterprise success and cultural context: the case of South Asia. Entrepreneurship Theory and Practice, 26(4), 131-143. doi: 10.1177/104225870202600408

Longino, H. (1990). Science as social knowledge: values and objectivity in scientific inquiry. Princeton University Press.

López, M., Lerendegui, M., Simon, T., Aznar,T., Martin, D., Briz, T. y

Botaya, R. (2011). Una mirada al género en la investigación. Análisis cualitativo. Atención primaria, 43(10), 531-535. doi:

10.1016/j.aprim.2010.09.018

Luque, B. (2008). El itinerario profesional de las mujeres jóvenes: una carrera de obstáculos. Anuario de Psicología, 39(1), 101-107

Nelson, L. (1990). Who Knows: From Quine to a Feministt Empiricism. Temple University Press: Philadelphia

Nieto, C. (2003). Mujeres empresarias y desarrollo rural. Experiencias en los municipios malagueños. (Tesis doctoral). Recuperado de https://www.researchgate.net/publication/43550089_Mujeres_empres arias_y_desarrollo_rural_Experiencias_en_los_municipios_malaguen os? ev=auth_pub

Rappaport, J. (1987). Terms of empowerment/exemplars of prevention:

Toward a theory for community psychology. American journal of community psychology, 15(2), 121-148.

Romero, M. (1996). El asesoramiento en educación. Granada: Aljibe. Ruiz-Olabuénaga, J. I. (2012). Metodología de la investigación cualitativa. Bilbao: Universidad de Deusto.

Ruiz-Olabuénaga, J. I. (2013). Teoría y práctica de la investigación cualitativa. Bilbao: Universidad de Deusto.

Steinmetz, G. (2005). The politics of method in the social sciences:

Positivism and its epistemological others. Durham: Duke University Press,

Taylor, J. y Bogdan, R. (2006). Introducción a los métodos cualitativos de investigación. Barcelona: Paidós.

Velaz de Medrano, C. (2003). Intervención educativa y orientadora en problemas de inadaptación y exclusión social por factores educativos y socioculturales. Madrid: UNED.

Velaz de Medrano, C. (2005). El asesoramiento en educación no formal: una mirada desde los orígenes de este modelo de intervención. En C. 
Monereo y J.L Pozo (Coords). La práctica del asesoramiento educativo a examen (pp. 119-138). Barcelona: Graó.

Villariño, M. y Cánnoves, G. (2000): 'Turismo rural en Galicia. Sin mujeres imposible'. En D. García-Ramón y M. Baylina. (Eds): El nuevo papel de las mujeres en el desarrollo rural (pp.171-198). Barcelona: Oikostau.

William, D. (2004). Effects of childcare activities on the duration of selfemployment in Europe. Entrepreneurship Theory \& Practice, 28(5), pp. 467-485. doi: 10.1111/j.1540-6520.2004.00058.x

Carmen de la Mata is Professor at University of Córdoba, Spain

Bárbara Luque is Professor at University of Zaragoza, Spain

Contact Address:m02maagm@uco.es 